International Journal of Pure and Applied Mathematics

Volume 83 No. 4 2013, 589-599

ISSN: 1311-8080 (printed version); ISSN: 1314-3395 (on-line version)

url: http://www.ijpam.eu

doi: http://dx.doi.org/10.12732/ijpam.v83i4.7

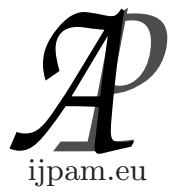

\title{
A LIMIT TO REPRESENT BERNOULLI NUMBERS USING EULERIAN NUMBERS
}

\author{
Mauri Aparecido de Oliveira ${ }^{1}$, Daniel Reed Bergmann ${ }^{2}$ \\ Luciana Massaro Onusic ${ }^{3}$ \\ ${ }^{1}$ Federal University of Sao Paulo - UNIFESP \\ ${ }^{2}$ University Nine of July - UNINOVE \\ ${ }^{3}$ Federal University of Sao Paulo - UNIFESP
}

\begin{abstract}
The Bernoulli Numbers are a sequence of rational numbers applied in various mathematics fields and can be written as a limit with $t$ tending toward zero applied to the nth derivative of the function $t /\left(e^{t}-1\right)$. From this result, the Bernoulli Numbers are written in terms of the Eulerian Numbers. From sucessives derivatives of the function $t /\left(e^{t}-1\right)$ the Eulers Triangle is obtained and a second triangle which allow to construct a limit to represent Bernoulli Numbers using Eulerian Numbers.
\end{abstract}

AMS Subject Classification: 11B68, 11B34, 11M99

Key Words: Bernoulli numbers, Bernoulli polynomials, Eulerian numbers, Euler's triangle

\section{Introduction}

In 1303 the chinese mathematician Zhu Shijie constructed a matrix of numbers, arranged in the shape of a triangle, from which one can draw several interesting numerical relations. Three hundred and fifty years after is published Traite $d u$ triangle arithmetique by Blaise Pascal, which again is treated exactly the same triangle of numbers discovered by Zhu Shijie. This triangle of numbers nowadays is better knowm as Pascal's Triangle. The work Katsuyo Sampo, due to the japanese mathematician Takakazu Seki Kowa, published posthumously

Received: November 10, 2012

(c) 2013 Academic Publications, Ltd.

$\S$ Correspondence author url: www.acadpubl.eu 
in the year 1712, presents a sequence of numbers that plays an important role in several mathematics branches. In 1713 the work Ars Conjectandi, due to the swiss mathematician Jacob Bernoulli, appears again the sequence of numbers discovered by Seki Kowa, in Chapter 3 can be find some of these numbers, written as $A=1 / 6, B=-1 / 30, C=1 / 42$ and $D=-1 / 30$.

The sequence of numbers discovered by Seki Kowa and Jacob Bernoulli is called Bernoulli Numbers, the first nine numbers are: $B_{0}=1, B_{1} \pm 1 / 2$, $B_{2}=1 / 6, B_{3}=0, B_{4}=-1 / 30, B_{5}=0, B_{6}=1 / 42, B_{7}=0$ and $B_{8}=-1 / 30$.

The Bernoulli Numbers can be obtained, for example, from Pascal's Triangle and one of its main applications refers to one of the greatest mathematical challenge of the seventeenth century, called "The Problem of Basel which was to determine the exact value of the infinite series:

$$
1+\frac{1}{4}+\frac{1}{9}+\frac{1}{25}+\cdots+\frac{1}{n^{2}}+\cdots .
$$

Leonhard Euler, presented a more general solution to this problem considering the case $\sum_{n=1}^{\infty} \frac{1}{n^{2 k}}$ and summarized in the expression

$$
\zeta(2 k)=\frac{(-1)^{k-1} 2^{2 k-1} \pi^{2 k}}{(2 k) !} B_{2 k} .
$$

Here $B_{2 k}$ exactly corresponds to the pairs Bernoulli Numbers.

\section{Analysis of Derivative $\frac{d^{n}}{d t^{n}} \frac{t}{e^{t}-1}$}

Definition 2.1. The Bernoulli polynomials comprise a sequence of polynomials $\left\{B_{n}(x)\right\}_{n=0}^{\infty}$, which have the following properties:

$$
\begin{gathered}
B_{0}(x)=1, \\
\frac{d}{d x} B_{n}(x)=n B_{n-1}(x), \\
\int_{0}^{1} B_{n}(x) d x=0, \quad \text { for } n \geq 1 .
\end{gathered}
$$

The numbers obtained for $B_{n}(0)$, with $n=0,1,2, \ldots$, are called Bernoulli Numbers. The generating functions commonly used to describe the the Bernoulli Numbers and the Bernoulli polynomials are respectively given by

$$
\sum_{n=0}^{\infty} B_{n} \frac{t^{n}}{n !}=\frac{t}{e^{t}-1}
$$


and

$$
\sum_{n=0}^{\infty} B_{n}(x) \frac{t^{n}}{n !}=\frac{t e^{t x}}{e^{t}-1} .
$$

To confirm the result (3) we can expand the right side using Taylor series, which yields:

$$
\begin{aligned}
\frac{t}{e^{t}-1} & =\lim _{a \rightarrow 0} \frac{a}{e^{a}-1}+\lim _{a \rightarrow 0}\left[\left(\frac{d}{d a} \frac{a}{e^{a}-1}\right) \frac{(t-a)}{1 !}\right] \\
+ & \lim _{a \rightarrow 0}\left[\left(\frac{d^{2}}{d a^{2}} \frac{a}{e^{a}-1}\right) \frac{(t-a)^{2}}{2 !}\right]+\lim _{a \rightarrow 0}\left[\left(\frac{d^{3}}{d a^{3}} \frac{a}{e^{a}-1}\right) \frac{(t-a)^{3}}{3 !}\right]+\cdots
\end{aligned}
$$

In the case where $a$ is equal to zero we have a MacLaurin series, but if we make $a$ tend to zero at $a /\left(e^{a}-1\right)$ we have an undetermined limit, which implies the use of the L'Hopital rule. Thus, we have that:

$$
\begin{gathered}
\lim _{a \rightarrow 0} \frac{a}{e^{a}-1}=1, \\
\lim _{a \rightarrow 0}\left(\frac{d}{d a} \frac{a}{e^{a}-1}\right)=-\frac{1}{2}, \\
\lim _{a \rightarrow 0}\left(\frac{d^{2}}{d a^{2}} \frac{a}{e^{a}-1}\right)=\frac{1}{6}, \\
\lim _{a \rightarrow 0}\left(\frac{d^{3}}{d a^{3}} \frac{a}{e^{a}-1}\right)=0, \\
\lim _{a \rightarrow 0}\left(\frac{d^{4}}{d a^{4}} \frac{a}{e^{a}-1}\right)=-\frac{1}{30} .
\end{gathered}
$$

With these results and the expression (3) we find:

$$
\sum_{n=0}^{\infty} B_{n} \frac{t^{n}}{n !}=B_{0} \frac{t^{0}}{0 !}+B_{1} \frac{t^{1}}{1 !}+B_{2} \frac{t^{2}}{2 !}+B_{3} \frac{t^{3}}{3 !}+B_{4} \frac{t^{4}}{4 !}+\ldots
$$

and knowing that

$$
\frac{t}{e^{t}-1}=1-\frac{1}{2} \frac{t}{1 !}+\frac{1}{6} \frac{t^{2}}{2 !}+0 \frac{t^{3}}{3 !}-\frac{1}{30} \frac{t^{4}}{4 !}+\ldots
$$

We can then conclude that the Bernoulli Numbers can be expressed using the following limit:

$$
B_{n}=\lim _{t \rightarrow 0}\left(\frac{d^{n}}{d t^{n}} \frac{t}{e^{t}-1}\right) .
$$


Figure 1 shows the behavior of some derivatives of the function $f(t)=$ $t /\left(e^{t}-1\right)$ near the Bernoulli Numbers. The first column of Figure 1 shows the function, the second column have the graphs shown in the interval $[-10,10]$ and the third column have been changed to reduced intervals to visualize the behavior of the function next to the $B_{n}$ corresponding. We can observe the fluctuation of functions representing those derivatives of $t /\left(e^{t}-1\right)$ near, respectively, to the three first Bernoulli Numbers $B_{0}=1, B_{1}=-1 / 2$ and $B_{2}=1 / 6$. Considering some of the derivatives of $t /\left(e^{t}-1\right)$ and rewriting the numerator such that we have first the terms containing $t e^{t}$ and then the terms containing $e^{t}$, we have the following results:

$$
\frac{d^{0}}{d t^{0}} \frac{t}{e^{t}-1}=\frac{t}{e^{t}-1}
$$

$$
\frac{d}{d t} \frac{t}{e^{t}-1}=\frac{-t e^{t}+e^{t}-1}{\left(e^{t}-1\right)^{2}}
$$

$$
\frac{d^{2}}{d t^{2}} \frac{t}{e^{t}-1}=\frac{t e^{2 t}+t e^{t}-2 e^{2 t}+2 e^{t}}{\left(e^{t}-1\right)^{3}}
$$

$$
\frac{d^{3}}{d t^{3}} \frac{t}{e^{t}-1}=\frac{-t e^{3 t}-4 t e^{2 t}-t e^{t}+3 e^{3 t}-3 e^{t}}{\left(e^{t}-1\right)^{4}}
$$

$$
\frac{d^{4}}{d t^{4}} \frac{t}{e^{t}-1}=\frac{t e^{4 t}+11 t e^{3 t}+11 t e^{2 t}+t e^{t}-4 e^{4 t}-12 e^{3 t}+12 e^{2 t}+4 e^{t}}{\left(e^{t}-1\right)^{5}}
$$

$$
\frac{d^{5}}{d t^{5}} \frac{t}{e^{t}-1}=\frac{-t e^{5 t}-26 t e^{4 t}-66 t e^{3 t}-26 t e^{2 t}-t e^{t}+5 e^{5 t}+50 e^{4 t}-50 e^{2 t}-5 e^{t}}{\left(e^{t}-1\right)^{6}}
$$




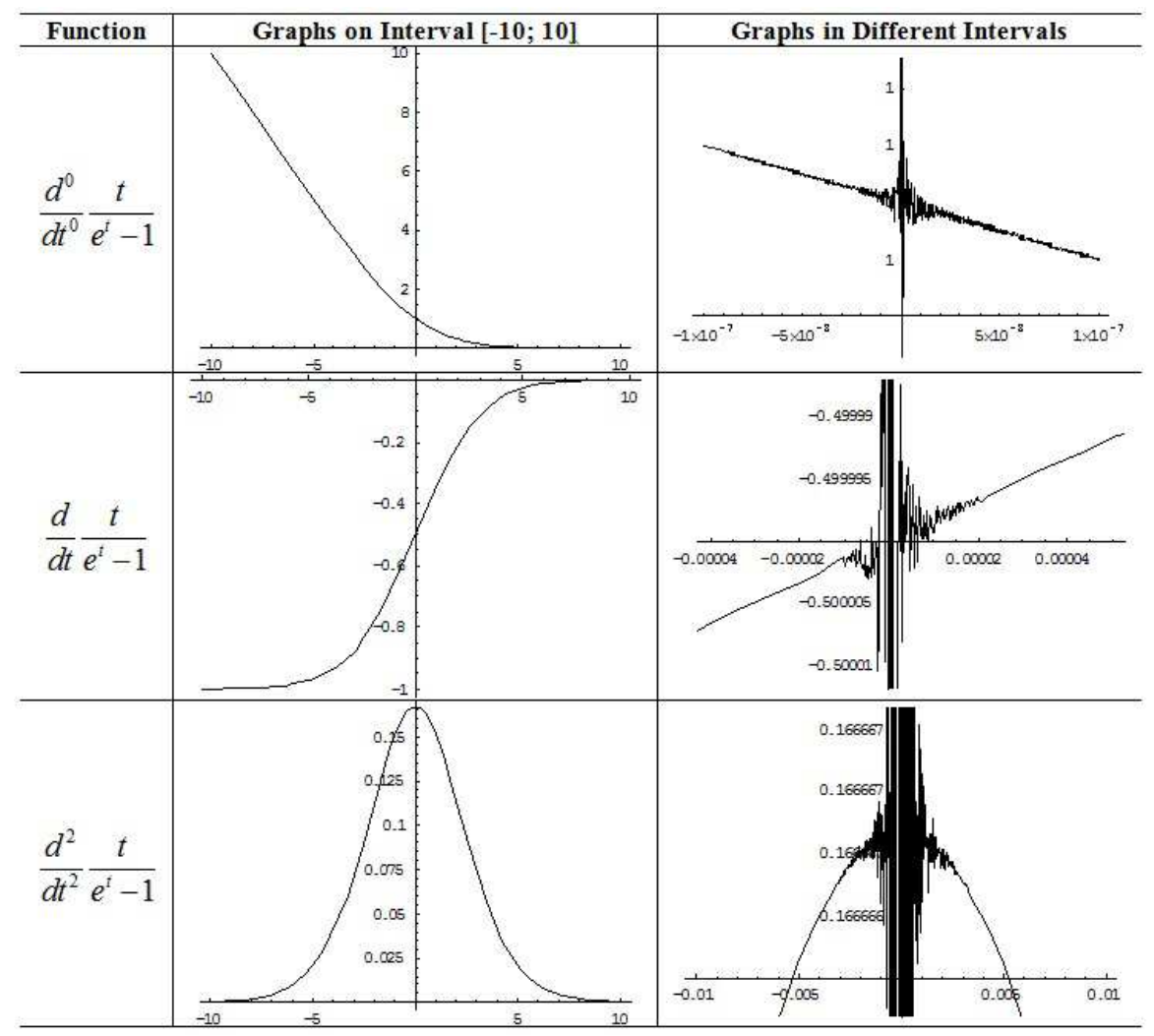

Figure 1: Graphs of derivatives of $t /\left(e^{t}-1\right)$ at different intervals.

We can observe that the nth derivative of the function $t /\left(e^{t}-1\right)$ can be represented as follows:

$$
\frac{d^{n}}{d t^{n}} \frac{t}{e^{t}-1}=\frac{\sum_{i=1}^{n} c_{i} t e^{n-(i-1) t}+\sum_{i=1}^{n} d_{i} e^{n-(i-1) t}}{\left(e^{t}-1\right)^{n+1}}
$$

Thus, we have the sum of the terms containing the product $t e^{n-(i-1) t}$ given by:

$$
\sum_{i=1}^{n} c_{i} t e^{n-(i-1) t}=c_{1} t e^{n t}+c_{2} t e^{(n-1) t}+c_{3} t e^{(n-2) t}+\ldots+c_{n} t e^{t}
$$

in the case that we have only exponential portions $e^{n-(i-1) t}$ the sum may be 
represented as:

$$
\sum_{i=1}^{n} d_{i} e^{n-(i-1) t}=d_{1} e^{n t}+d_{2} e^{(n-1) t}+d_{3} e^{(n-2) t}+\ldots+d_{n} e^{t}
$$

From the results obtained, can be seen that in the case of $c_{i}$ coefficients, we have the following relations:

$$
\begin{aligned}
& \left|c_{1}\right|=\left|c_{n}\right|=1, \\
& \left|c_{2}\right|=\left|c_{n-1}\right|, \\
& \left|c_{3}\right|=\left|c_{n-2}\right|,
\end{aligned}
$$

Here $-c_{i}$ - describes the absolute value of the coefficient $c_{i}$.

In the first derivative of $t /\left(e^{t}-1\right)$ the numerator is equal to $-t e^{t}+e^{t}-1$ and is the only case in which we have a constant, in this case -1 , which is not multiplied by $e^{n-(i-1) t}$ or $t e^{n-(i-1) t}$ where $n-(i-1) \neq 0$. This symmetry of values can also be observed in the case of coefficients $d_{i}$, allowing writing when $n \geq 3$, that:

$$
\begin{aligned}
& \left|d_{1}\right|=\left|d_{n}\right|=n, \\
& \left|d_{2}\right|=\left|d_{n-1}\right|, \\
& \left|d_{3}\right|=\left|d_{n-2}\right|,
\end{aligned}
$$

Here $-d_{i}$ - describes the absolute value of the coefficient $d_{i}$.

When the order $n$ of the derivative is odd and $n \geq 3$ we can verify that $d_{\frac{n+1}{2}}=0$. Another feature that can also be extract is the fact that $d_{i}$ is multiple of $n$.

From the derivatives of of $t /\left(e^{t}-1\right)$ we can construct a matrix containing only the signals, obtained from each of the portions of the numerators, shown in Table 1.

In Table 1, the first columns shows the order of the derivative of $t /\left(e^{t}-1\right)$, lines contain only the signals of the numerators, for example, the fourth-order derivative is:

$$
\left(t e^{4 t}+11 t e^{3 t}+11 t e^{2 t}+t e^{t}-4 e^{4 t}-12 e^{3 t}+12 e^{2 t}+4 e^{t}\right) /\left(e^{t}-1\right)^{5}
$$




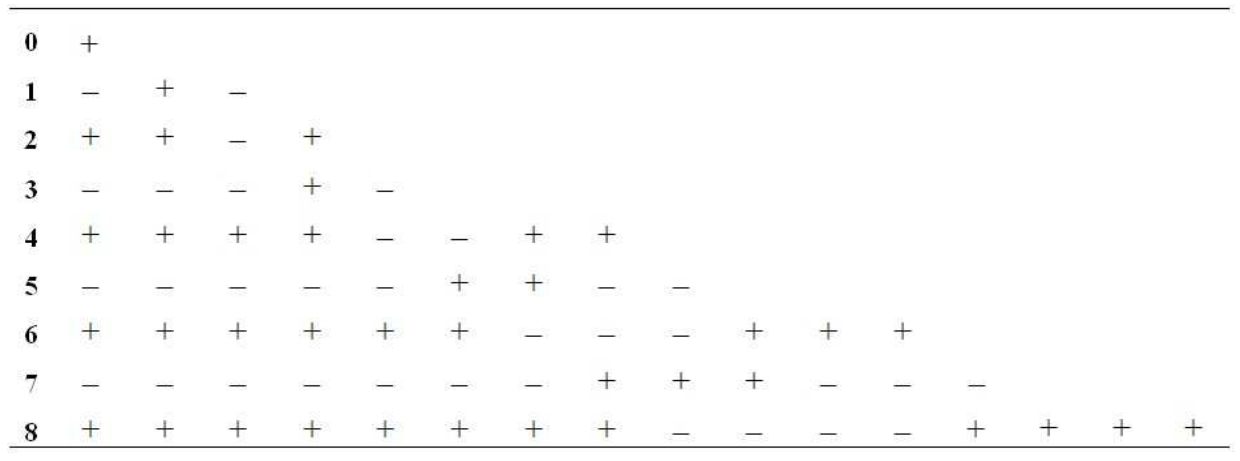

Table 1: Matrix of signals of the numerator coefficients in the derivatives of $t /\left(e^{t}-1\right)$

such that the signals of the coefficients $c_{i}$ and $d_{i}$, are given by,,,,,,++++--+ and + . On the line that corresponds to the first derivative, the last signal belongs to the constant -1 . The signals from the numerators of the coefficients obtained from derivatives of the function $t /\left(e^{t}-1\right)$, can be obtained for $n \geq 2$ using the expression:

$$
\sum_{i=1}^{n}(-1)^{n}\left[t+\operatorname{sgn}\left(\frac{n+1}{2}-i\right)\right] e^{i t},
$$

being $\operatorname{sgn}(x)$ the sign function given by:

$$
\operatorname{sgn}(x)=\left\{\begin{array}{cc}
1, & x>0 \\
0, & x=0 \\
-1, & x<0
\end{array}\right.
$$

Using the steps above and knowing that the sequence of signals for the coefficients $c_{i}^{(n)}$ and $d_{i}^{(n)}$ can be obtained from the expression (11), with results presented in Tables 2 and 3. The numbers presented in Table 2 are called Eulerian Numbers and the matrix formed by these numbers is called Euler's Triangle. A numeric matrix presented in Table 3 is built from the Euler's Triangle.

In Tables 2 and 3 we have that $\left|c_{i}^{(n)}\right|$ and $\left|d_{i}^{(n)}\right|$ represents, respectively, the absolute value of the coefficient $c_{i}$ and $d_{i}$ from the nth derivative of the function $t /\left(e^{t}-1\right)$. 


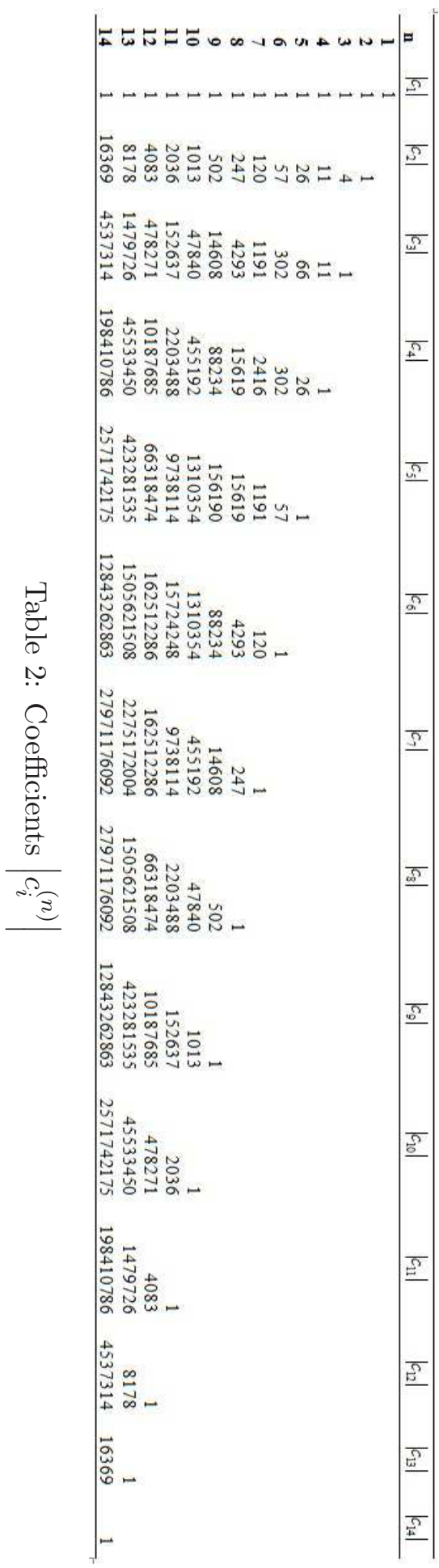




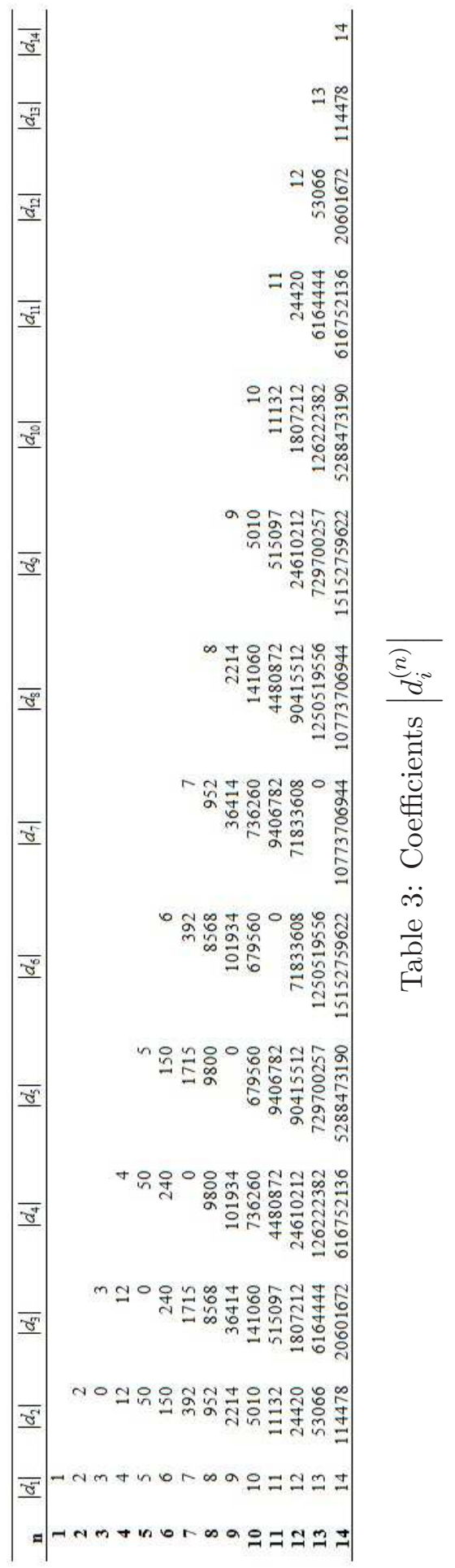




\section{Limit Representation of $B_{n}$ using $E_{n, m}$}

Definition 3.1. The numbers $E_{n, m}$ of orderings $(1,2,3, \ldots, n)$ with increasing $m$ sequences are called Eulerian Numbers and obtained by the expresion:

$$
E_{n, m}=\sum_{k=1}^{m}(-1)^{m-k}\left(\begin{array}{c}
n+1 \\
m-k
\end{array}\right) k^{n}, m=1,2,3, \ldots
$$

Proposition 3.1. Let $B_{n}$ a Bernoulli Number and $E_{n, m}$ a Eulerian Number, we have that $B_{n}$ can be represented from $E_{n, m}$ through the following limit:

$$
B_{n}=\lim _{t \rightarrow 0} \frac{\sum_{i=1}^{n}(-1)^{n}\left[t E_{n, i}+n\left(E_{n-1, i}-E_{n-1, i-1}\right)\right] e^{i t}}{\left(e^{t}-1\right)^{n+1}}, n>1 .
$$

Proof. From the results obtained in the previous section we can write the nth derivative of the function $t /\left(e^{t}-1\right)$ as follows:

$$
\frac{d^{n}}{d t^{n}} \frac{t}{e^{t}-1}=\frac{\sum_{i=1}^{n}(-1)^{n}\left[c_{i} t+d_{i} \operatorname{sgn}\left(\frac{n+1}{2}-i\right)\right] e^{i t}}{\left(e^{t}-1\right)^{n+1}}
$$

knowing that in expression (12) when $m$ is zero we have $E_{n, 0}=0$ and in (14) making the substitutions

$$
c_{i}=E_{n, i}
$$

and

$$
d_{i} \operatorname{sgn}\left(\frac{n+1}{2}-i\right)=n\left(E_{n-1, i}-E_{n-1, i-1}\right),
$$

enables the construction of the following expression for the nth derivative of the function $t /\left(e^{t}-1\right)$ :

$$
\frac{d^{n}}{d t^{n}} \frac{t}{e^{t}-1}=\frac{\sum_{i=1}^{n}(-1)^{n}\left[t E_{n, i}+n\left(E_{n-1, i}-E_{n-1, i-1}\right)\right] e^{i t}}{\left(e^{t}-1\right)^{n+1}}, n>1
$$

from where the result (13) follows.

Example. Consider the Bernoulli number $B_{14}$ which is equal to $7 / 6$, using the expression (13) we have the following result:

$$
\begin{aligned}
B_{14}=\lim _{t \rightarrow 0} & \frac{\sum_{i=1}^{14}(-1)^{14}\left[t E_{14, i}+n\left(E_{14-1, i}-E_{14-1, i-1}\right)\right] e^{i t}}{\left(e^{t}-1\right)^{14+1}} \\
& =\lim _{t \rightarrow 0} \frac{\sum_{i=1}^{14}(-1)^{14}\left[t \sum_{k=1}^{m}(-1)^{m-k}\left(\begin{array}{c}
14+1 \\
m-k
\end{array}\right) k^{14}+\right.}{\left(e^{t}-1\right)^{14+1}}
\end{aligned}
$$




$$
\begin{array}{r}
\frac{+14\left(\sum_{k=1}^{m}(-1)^{m-k}\left(\begin{array}{c}
14-1+1 \\
m-k
\end{array}\right) k^{14-1}+\right.}{\left(e^{t}-1\right)^{14+1}} \\
\frac{\left.\left.-\sum_{k=1}^{m}(-1)^{m-1-k}\left(\begin{array}{c}
14-1+1 \\
m-1-k
\end{array}\right) k^{14-1}\right)\right] e^{i t}}{\left(e^{t}-1\right)^{14+1}} .
\end{array}
$$

yielding $B_{14}=\frac{7}{6}$.

\section{References}

[1] T. Kim, Euler numbers and polynomials associated with zeta functions, Abstract and Applied Analysis, Article ID 581582, 11 pages (2008).

[2] Q.M. Luo, F. Qi, L. Debnath, Generalizations of Euler numbers and polynomials, IJMMS, 2003, No. 61 (2003), 3893-3901.

[3] D. Foata, Eulerian polynomials: From Euler's time to the present, The legacy of Alladi Ramakrishnan in the Mathematical Sciences, 253273, Springer, New York (2010).

[4] L. Carlitz, Eulerian numbers and polynomials, Mathematics Magazine, 32, No. 5 (1959), 247-260.

[5] M.A. Oliveira, R.H. Ikeda, Representation of the nth Derivative of the Normal PDF Using Bernoulli Numbers and Gamma Function, Applied Mathematical Sciences, 6, No. 74 (2012), 3661-3673.

[6] K.W. Chen, M.A. Eie, Note on generalized Bernoulli numbers, Pacific Journal of Mathematics, 199, No. 1 (2001).

[7] H. Cohen, Number Theory, Volume II: Analytic and Modern Tools, Graduate Texts in Mathematics, Springer (2007).

[8] T.M. Apostol, Introduction to Analytic Number Theory, Springer-Verlag, New YorkHeidelbergBerlin (1976).

[9] F. Chung, R. Graham, D. Knuth, A symetrical Eulerian identity, Journal of Combinatorics, 17, No. 1 (2010), 29-38.

[10] H.W. Gould, Explicit formulas for Bernoulli numbers, The American Mathematical Monthly, 79, No. 1 (1972), 44-51. 
